André Le Roux

Centre de recherche en gestion

Université de Poitiers

F-86000

aleroux@poitiers.iae-france.fr

Marinette Thébault

Centre de recherche en gestion

Université de Poitiers

F-86000

mthebault@poitiers.iae-france.fr

\title{
Territoire et territoire numérique de la résistance des consommateurs
}

\section{Une étude exploratoire}

Résumé. - Concept proposé pour qualifier les comportements de défiance, d'opposition voire de rébellion, la résistance du consommateur aux actions et dispositifs marketing est affectée par le développement du numérique. Source d'information, le numérique constitue aussi un nouveau territoire d'expression, de pratiques et d'usages. L'article explore la notion de territoire de résistance du point de vue du consommateur en étudiant la catégorisation d'une pluralité de comportements. La démarche conduit à explorer le territoire thématique de la résistance, à l'aide d'une étude quantitative par questionnaire. Trois territoires sont mis en évidence : un territoire de la résistance, un territoire de la non-résistance et un territoire ambivalent. L'hypothèse d'une césure générationnelle est également interrogée. Ces résultats sont discutés à la lumière des concepts de territoire, de territoire numérique et de leurs implications pour les acteurs de marché.

Mots clés. - résistance du consommateur, actions et dispositifs marketing, territoire, territoire numérique, étude quantitative, digital natives 


\section{André Le Roux, Marinette Thébault, Consumers' Resistance Territory and Digital Territory. An Exploratory Research}

Consumer resistance to marketing actions and devices a concept proposed in order to qualify defiance, opposition or even rebellion behaviors, is affected by the development of digital activities. A source of information, digital is also a new territory of expression, practices and usages. This research explores the concept of resistance territory from the consumer standpoint through studying categorization of various behaviors, thanks to a quantitative survey. Results highlight three territories: a resistance territory, a non-resistance territory, an ambivalent territory, and investigates the hypothesis of a generation gap. Results are discussed in light of the concepts of territory, digital territory and of their implications for market actors.

Keywords : consumer resistance, marketing actions and devices, territory, digital territory, quantitative survey, digital natives 
La notion de résistance du consommateur a émergé progressivement dans la littérature marketing pour constituer un courant de recherche significatif (Hirschman, 1970 ; Peñaloza, Price, 1993 ; Roux, 2009). Parallèlement, le numérique est devenu une réalité majeure à laquelle le consommateur est confronté dans ses comportements et ses usages. Source d'information, mais aussi espace d'expression, le numérique constitue un champ d'action nouveau et évolutif pour le consommateur, un nouveau territoire d'expression, de pratiques et d'usages. La recherche dont est issu cet article se situe donc à la croisée de deux courants de recherche issus de disciplines différentes : le courant issu du marketing sur la résistance du consommateur aux actions et dispositifs marketing ; celui issu des sciences de l'information et de la communication sur les concepts de territoires et de territoires numériques. Elle tend à explorer la notion de territoire de résistance du consommateur aux actions et dispositifs marketing. Elle étudie une pluralité de comportements considérés comme résistants dans la littérature, en demandant aux consommateurs de catégoriser chacun des comportements examinés, afin de mettre en évidence le territoire thématique de la résistance. Un autre objectif est d'explorer la dimension numérique du territoire de la résistance, du point de vue du champ d'action couvert, mais aussi du point de vue des modifications apportées par le numérique sur les actions de résistance et les acteurs de la résistance. Il s'agit d'examiner notamment l'existence d'une césure générationnelle fondée sur le numérique. Ainsi revenonsnous sur l'hypothèse des digital natives (Prensky, 2001a, 2001b ; Palfrey, Gasser, 2008) dont on connaît l'intérêt et la fragilité (Thomas, 2011 ; Jones, Shao, 2011 ; Stenger, 2015) pour le comportement du consommateur en particulier (Le Roux, Stenger, Thébault, 2015 ; Stenger, 2015).

Après avoir défini les notions de résistance du consommateur aux actions et dispositifs marketing, de territoire et de territoire numérique, nous présentons les résultats d'une étude 
quantitative exploratoire réalisée auprès de consommateurs. Ceux-ci ont été interrogés sur leur catégorisation de pratiques de résistance aux actions et dispositifs marketing. Ces résultats sont discutés à la lumière des concepts de territoire et de territoire numérique, et de leurs implications pour les acteurs de marché.

\section{La résistance du consommateur aux actions et dispositifs marketing}

Étudier le territoire de la résistance nécessite d'abord de définir le concept de résistance du consommateur. Celui-ci a été proposé par Lisa Peñaloza et Linda L. Price (1993) et Robert O. Herrmann (1993) pour qualifier les comportements de défiance, d'opposition voire de rébellion du consommateur. Il trouve son origine dans les travaux d'Albert O. Hirschman (1970) qui, outre la fidélité («loyalty»), identifie le pouvoir d'expression («voice») et de défection («exit») du consommateur. Pour sa part, Dominique Roux (2009: 131) définit la résistance comme «un état motivationnel qui pousse le consommateur à s'opposer à des pratiques, des logiques ou des discours marchands jugés dissonants ».

La notion de résistance suppose la perception d'une force exercée sur le consommateur, sa catégorisation comme inacceptable et l'intentionnalité de l'acteur à résister. Lionel Sitz (2009) précise également que la résistance peut être définie selon deux perspectives : une première perspective «actionnaliste» considère les conséquences du comportement considéré ; une seconde perspective «intentionnaliste » définit la résistance par l'intention consciente de l'individu à résister. Par ailleurs, la qualification de comportements de consommateurs comme résistants peut être établie par un observateur «extérieur» (chercheur, responsable marketing...) ou par le consommateur lui-même. La première 
approche, qualifiée d' «etic », est celle retenue par la sociologie critique et, le plus souvent, par le marketing. Dite «emic », la seconde connaît un intérêt croissant (Roux, 2014). Les deux points de vue ne se recouvrent pas totalement. Un comportement pourra être qualifié de résistant par un observateur extérieur sans que le consommateur ait l'intention de résister (perception erronée) tandis qu'une action intentionnellement résistante mais individuelle et isolée échappera à l'observateur extérieur et ne sera pas catégorisée comme résistante par ce dernier (résistance non perçue volontaire).

Sur le plan théorique, la résistance du consommateur est un construit multidimensionnel. Susan Fournier (1998) la conçoit comme un continuum de comportements et d'activités allant de réactions d'évitement jusqu'à des formes plus radicales de rébellion active telles la plainte, le boycott ou l'abandon en passant par des stratégies d'ajustement ou de restriction volontaire. Lisa Peñaloza et Linda L. Price (1993) définissent les comportements de résistance en fonction de quatre axes: comportements collectifs versus individuels; comportements réformistes versus radicaux ; comportements dirigés contre les offres versus contre les signes véhiculés par les firmes ; comportements internes versus externes aux institutions marketing. Dominique Roux (2007) distingue la résistance ciblée, contre les signes, les discours, les dispositifs et les comportements des firmes, de la résistance globale, contre le fonctionnement et les logiques du marché (simplicité volontaire, consommation verte...).

Les recherches sur les comportements étudiés au titre de la résistance sont nombreuses et diverses. Elles ont porté sur le boycott (Friedman, 1985 ; Thébault, 1999 ; Cissé-Depardon, 2009 ; Cissé-Depardon, N’Goala, 2009 ; Capelli, Legrand, Sabadie, 2012), sur la résistance aux marques (Thompson, Haytko, 1997 ; Duke, 2002 ; Moisio, Askegaard, 2002 ; Dalli, Gistri, Romani, 2005), à la publicité (Rumbo, 2002 ; Cottet, Ferandi, Lichtle, 2009, 2012), au 
placement des marques dans les films (Fontaine, 2009), aux techniques de vente (Kirmani, Campbell, 2004 ; Roux, 2007, 2008), aux programmes de fidélité (Pez, 2012) et sur les plaintes et les réclamations (Roux, 2012). D'autres recherches se sont centrées sur la résistance à certaines formes de marché par la mise en place de pratiques alternatives d'échanges (Robert-Demontrond, 2009), la résistance aux dons d'argent (Urbain, Gall-Ely, Urien, 2012), le rejet de la consommation et la simplicité volontaire (Dobscha, Ozanne, 2001 ; Shaw, Newholm, 2002 ; Zavestoski, 2002).

Le champ des activités numériques est assez peu étudié comparativement au boycott ou à la résistance à la publicité. Toutefois, on peut remarquer les recherches sur les comportements de copie et de téléchargement illégal, le piratage et le hacking ${ }^{1}$ (Cox, Collins, Drinkwater, 2010 ; Garcia-Bardidia, Nau Rémy, 2011 ; 2012 ; Kerr et al., 2012 ; Guiton, 2013 ; Odou, Bonnin, 2014), la cyber-résistance (Chalamon, Chouk, Guiot, 2012), ainsi que les comportements d' «anti-branding » sur l'internet (Krishnamurthy, Kucuk, 2009), d'évitement de la publicité sur l'internet (Cho, Cheon, 2004) ou sur les réseaux sociaux numériques en particulier (Kelly, Kerr, Drenan, 2010).

Malgré l'abondance des sujets traités et des domaines abordés, les recherches sur la résistance souffrent de certaines limites. Il s'agit d'un champ dominé par des approches qualitatives, caractérisé par l'absence de mesures (Roux, 2007). De plus, du fait de ce type d'approche, un comportement ou une pratique est étudié(e) de manière approfondie et située culturellement et

\footnotetext{
${ }^{1}$ Le terme hacking est ici utilisé dans son acception grand public qui correspond à un « ensemble de techniques permettant d'exploiter les failles et vulnérabilités d'un élément ou d'un groupe d'éléments matériels ou humains ». Accès : https://fr.wikipedia.org/wiki/Hacking. Consulté le 07/01/2019. Le hacking est proche du piratage et se confond partiellement avec ce dernier lorsqu'il s'agit de piratage informatique sous la forme d'une intrusion illicite dans des réseaux informatiques. Le piratage est néanmoins plus large que le hacking lorsqu'il se manifeste sous la forme de cassage de codes de protection de logiciels (cracking), d'usurpation d'identité ou de hameçonnage.
} 
socialement, mais isolé(e) des autres pratiques et comportements de résistance que le consommateur peut adopter. En outre, si la fréquence des comportements de résistance a été étudiée, notamment chez les jeunes qualifiés de digital natives (Le Roux, Stenger, Thébault, 2015), la catégorisation des comportements ou pratiques de résistance par le consommateur lui-même n'a pas été abordée.

\section{Territoire et territoire numérique}

La notion de territoire a été peu explorée en marketing. En revanche, elle a été plus spécifiquement étudiée dans d'autres disciplines : bien sûr en géographie et, dans une moindre mesure, en sciences de l'information et de la communication.

Le territoire est rattaché à une entité physique. Il recouvre une réalité physique, symbolisée par les infrastructures nécessaires à son fonctionnement. Le territoire renvoie aussi à la notion de centralisation, une réalité qui oppose un centre à une périphérie, une configuration liée aux infrastructures conçues comme un système nerveux. De plus, le territoire se définit comme un espace conquis par une autorité, un État, une collectivité locale, une administration (Alliès, 1980). On parle de territoire national, régional. Dans ce territoire, l'autorité exerce son pouvoir, sa régulation. Il s'agit d'une construction juridique singulière construite historiquement.

Cependant, la carte n'est pas le territoire, l'infrastructure ne peut à elle seule rendre compte de la réalité du réseau, de ses pratiques : «Car si un certain équipement en est la condition, ce n'est pas l'autoroute de l'information entre deux villes du territoire qui devient essentielle 
mais la dynamique d'une communauté connectée, qui peut fort bien s'affranchir des contraintes de localisation » (Boullier, 2009 : 2). Cela renvoie à un ensemble de pratiques, de valeurs et à un monde beaucoup plus flou et mal connu. La notion de territoire thématique est pertinente pour penser et analyser les comportements et les pratiques des individus sur les réseaux. Comme le remarque Romain Badouard (2013 : 109), «les publics se réunissent dans des lieux, des arènes ${ }^{2}$ en ligne (des groupes, des réseaux sociaux, des sites, des listes de diffusion) en fonction de centres d'intérêt qu'ils partagent, et c'est à partir de ces arènes qu'ils se mobilisent pour conduire une action sur une scène publique ».

Le territoire numérique recouvre ces deux réalités, une réalité physique qui correspond aux infrastructures et aux réseaux nécessaires à son fonctionnement, mais aussi sociale avec des pratiques et des usages des individus dans les espaces, des représentations sociales et des valeurs. Dominique Boullier (2009) reprend cette dualité en distinguant les notions de territoires équipés, correspondant à la dimension infrastructure du territoire numérique, conçu comme un système nerveux, qualifié de topologie centralisée, et la notion de milieu associé, écosystème comprenant les échanges à l'intérieur du réseau mais aussi avec son environnement. Pierre Musso (2008: 6) oppose «la "tuyauterie" des réseaux» au «seul territoire réellement "numérique" : le cyberespace, dont Internet est la composante la plus visible, et les systèmes d'information des entreprises et des organisations, la plus stratégique ».

Cependant, l'adjonction du caractère numérique à la notion de territoire transforme profondément ce dernier. Le numérique étend les frontières du territoire non seulement du

\footnotetext{
${ }^{2}$ Sur les arènes publiques, voir D. Cefaï (2016).
} 
point de vue physique ou géographique, mais aussi du point de vue de sa nature et de la logique sous-jacente. Pierre Musso (ibid. : 33) propose la notion de territoire augmenté, ou hyperterritoire, qui « doit être comprise de manière à la fois extensive (territoire étendu) et intensive (intensification des capacités du territoire et de ses résidents). Le territoire est "augmenté" quand les capacités des personnes, des entreprises et autres institutions se trouvent amplifiées par des ressources auxquelles on accède via le réseau : informations, outils, applications, services ». Avec le numérique, le territoire, d'entité géographique située, se transforme en un monde parallèle où peuvent s'exprimer des comportements réunis plus par affinité que par proximité géographique. Indifférence à la distance (Musso, 2009) et affranchissement des contraintes de localisation (Boullier, 2009) caractérisent les résidents de ce territoire, qui bénéficient de capacités d'échanges et d'actions accrues. Pierre Musso (2008: 35) utilise le concept de cyberespace pour intégrer cette transformation. Le cyberespace est à la fois un espace de l'information, ce qui recouvre le contenu échangé via l'ensemble des systèmes d'information planétaires et son caractère immatériel, et un espace multiforme de rencontres et de confrontations de points de vue, de valeurs, un ensemble d'actions et de pratiques. Les distances ne sont plus physiques, mais «sociales, symboliques et mentales ».

Ce faisant, le territoire numérique devient a-territorial. Initialement conçu comme un espace conquis par une autorité, à travers le numérique, le territoire s'affranchit de sa dimension physique, donc de l'autorité régulatrice. D'un territoire conçu comme un système nerveux, une topologie centralisée, on passe avec le numérique à des formats d'association qui font émerger des «quasi-sujets» selon le concept de Michel Serres (1997). L'organisme centralisateur perd la maîtrise du réseau. Dominique Boullier (2009 : 10) fait référence à « des modes, des rumeurs, du $b u z z, \ldots$ qui vivent de leur vie, prolifèrent et dépassent de supposées 
intentions individuelles ou stratégiques ». Il pointe le caractère crucial des activités de veille pour tout gouvernement de territoires politiques ou d'entreprises qui devraient se consacrer à « ce zonage pertinent de capteurs d'alerte».

C'est ainsi que Fabien Granjon (2017: 9) distingue territoire de gouvernement et zone d'alerte. Le territoire de gouvernement renvoie à une conception centralisée du pouvoir avec les élus et porte-parole désignés par le peuple, tandis que la zone d'alerte renvoie à une réalité beaucoup plus diffuse liée à des anonymes que l'auteur appelle «sans voix»: «La prolifération des voix, des ci-devant "sans voix" comme on parlait des sans culottes, n'est pas un travers du numérique en réseaux, il en est constitutif techniquement par son architecture et par son extraterritorialité qui veut dire extra autorité ».

\section{Territoire et territoire numérique de la résistance du consommateur}

L'objectif de cette recherche est d'identifier le territoire de la résistance des consommateurs. Pour ce faire, il s'agit d'étudier une pluralité de comportements considérés comme résistants dans la littérature, en demandant aux consommateurs de catégoriser chacun des comportements examinés comme étant ou non de la résistance. Explorer les pratiques catégorisées comme résistantes par les consommateurs eux-mêmes permettra de mettre en évidence le territoire thématique de la résistance.

La dimension numérique du territoire de la résistance sera également prise en compte. Comme le remarque Fabien Granjon (2017 : 14-15), les territoires numériques sont un champ d'action naturel d'expression et de développement des mouvements protestataires, dont «la 
plupart partage le fait de dénoncer la démocratie représentative (désobéissance civile, valorisation du consensus, de la démocratie directe, etc.), mais aussi d'utiliser les technologies numériques d'information et de communication à des fins de diffusion, de débat, de mobilisation et de coordination $»$. Du fait de son affranchissement des contraintes géographiques ou de localisation, et par sa dimension affinitaire, le territoire numérique constitue un lieu virtuel propice à l'expression de dissensions, à l'organisation d'actions de protestation ou de contestation. Il offre aux consommateurs de nouvelles opportunités et possibilités de résistance.

En effet, le numérique étend les frontières du territoire de résistance du point de vue géographique. En tant que récepteur, l'individu peut plus facilement être informé, sensibilisé et prendre conscience de situations ou d'actions qu'il juge inacceptables et auxquelles il voudra résister. Ainsi le numérique facilite-t-il la mobilisation de mouvements de masse en permettant de joindre des individus initialement isolés géographiquement et idéologiquement. En tant qu'émetteur, l'individu accède à un territoire nouveau dont les frontières et les limites sont étendues. Par exemple, le bouche-à-oreille, qui auparavant était limité à un entourage proche, prend une nouvelle dimension avec les réseaux sociaux numériques. On passe d'un territoire limité physiquement, l'entourage, à un territoire étendu aux dimensions plus larges d'un point de vue extensif, mais aussi intensif avec des réponses et des comportements nouveaux comme les partages et les retweets.

En outre, le numérique redéfinit la nature de certains modes de résistance en rendant possible ou en facilitant et en donnant plus d'ampleur à certains comportements. Si les comportements alternatifs ou participatifs de troc, d'association pour le maintien de l'agriculture paysanne (Amap), d'échanges de services existaient bien avant le numérique, ce dernier leur fournit une 
possibilité de développement et de coordination sans comparaison avec un réseau non numérique.

Le numérique a aussi rendu possibles certaines activités. Le mouvement du logiciel libre n’a pu apparaître qu'avec la création de réseaux numériques. Les comportements de piratage et de téléchargement illégaux ne peuvent s'exprimer qu'à travers des réseaux numériques :

\footnotetext{
«Davantage qu'une simple infrastructure, fut elle "de résistance", Internet tend à redéfinir à la marge, mais aussi de plus en plus centralement, les frontières de l'action collective et la nature des modes de résistance et de contestation. Dès la fin des années 1990 et l'émergence du mouvement altermondialiste, le développement des usages d'Internet a permis que s'actualisent de nouveaux potentiels d'entraide, d'échange d'information, d'expertise et que soit facilitée l'articulation de fronts de contestation au niveau local, national et/ou international »(Granjon, $2017:$ 14-15).
}

Cette nouvelle situation justifie d'autant plus qu'on s'intéresse au territoire numérique de la résistance afin de prendre en compte cette réalité nouvelle, mais aussi cette opacité nouvelle et ses enjeux. En effet, le numérique donne au consommateur un accès direct à des modes de résistance individuels ou collectifs sans avoir à passer par des intermédiaires, des médiateurs, des représentants ou des filtres. Aujourd'hui, des actions telles le boycott, la plainte, la protestation ou le logiciel libre peuvent se développer sans avoir à passer par une association de consommateurs, de défense des droits de l'homme ou autre. Le territoire numérique de la résistance devient une zone d'extra-autorité. Il n'y a pas nécessairement un acteur qui lance un mot d'ordre de résistance, qui part d'un centre vers une périphérie. Les comportements de résistance s'inscrivent dans des phénomènes de contagion difficiles à anticiper, détecter et gérer.

La notion de résistance du consommateur aux actions et aux dispositifs marketing présente donc une forte convergence, et même une affinité, avec celle de territoire et avec le numérique 
considéré non seulement comme un territoire d'expression, mais aussi comme un environnement qui modifie profondément les pratiques et la notion de résistance.

L'objectif de cette recherche est d'explorer le territoire de la résistance aux actions et dispositifs marketing tel que les consommateurs le définissent : quelles sont les pratiques et actions que les consommateurs catégorisent comme résistantes par opposition à celles qu'ils ne considèrent pas comme telles ? Un autre objectif est l'étude de la dimension numérique du territoire de la résistance, du point de vue du champ d'action de la résistance mais aussi des modifications engendrées par le numérique au niveau des actions et des acteurs de la résistance. Est-ce que tous les consommateurs délimitent le territoire de résistance de la même façon ou, au contraire, le numérique introduit-il des clivages entre consommateurs ? Nous pouvons notamment poser la question d'une rupture générationnelle pour les pratiques numériques, popularisée à travers la métaphore des digital natives (Prensky, 2001a, 2001b ; Palfrey, Gasser, 2008 ; Prensky, 2015 ; Stenger, 2015).

\section{Une catégorisation de la résistance par les consommateurs eux-mêmes}

Ici, nous retenons une perspective intentionnaliste et cherchons à tendre vers une approche plus emic en proposant aux consommateurs de définir et catégoriser les pratiques comme constituant ou non de la résistance. La démarche n'est pas purement emic puisque les consommateurs interrogés se prononcent sur un ensemble de comportements et de pratiques établi par les auteurs (en reprenant notamment la littérature). Il ne s'agit pas de faire émerger des catégories à partir de discours et représentations indigènes (Olivier De Sardan, 1998). La 
démarche se veut moins etic qu'à l'accoutumée et plus emic en établissant une catégorisation des pratiques de résistance du point de vue du consommateur.

L'étude a été réalisée grâce à un questionnaire administré en face-à-face auprès d'un échantillon de convenance de 290 individus $^{3}$. L'échantillon comporte $48 \%$ d'hommes et $52 \%$ de femmes. Il est composé d'individus de 17 à 74 ans, avec un âge moyen de 31,76 ans (écart type : 13,25). Sur le plan des catégories socioprofessionnelles, $50 \%$ sont étudiants et $47 \%$ actifs. Pour examiner une éventuelle césure générationnelle et, plus spécifiquement, les pratiques des «jeunes nés avec le numérique », la notion de digital natives est la plus couramment utilisée. Celle-ci a été proposée et opérationnalisée par Marc Prensky (2001a, 2001b, 2009, 2010) grâce à l'âge des répondants. Les digital natives ont été définis comme les individus de 17 à 30 ans au moment de l'étude tandis que les plus de 30 ans ont été catégorisés en tant qu'«aînés ». En conséquence, dans cette étude, les répondants ont été scindés en deux catégories : les digital natives, âgés de 17 à 30 ans, face à leurs aînés, âgés de plus de 30 ans.

Le questionnaire comporte 25 comportements qualifiés de résistants dans la littérature. Ces comportements ou pratiques ont été choisis après examen critique de la littérature. Nous avons considéré les deux premiers axes définis par Lisa Peñaloza et Linda L. Price (1993) en incluant des comportements individuels et collectifs (plainte individuelle versus collective, piratage individuel versus en réseau), réformistes et radicaux (plainte versus boycott, installation et développement de logiciels libres versus hacking et piratage). Nous avons

\footnotetext{
${ }^{3}$ L'étude a été réalisée dans le cadre d'un module d'initiation à la recherche au sein d'un master en marketing. Les étudiants devaient administrer 10 questionnaires dans leur entourage en interrogeant 5 personnes ayant un statut étudiant et 5 personnes non étudiant. Les questionnaires ont été administrés du 15 décembre 2012 au 10 janvier 2013.
} 
également pris en compte la distinction apportée par Dominique Roux (2007) entre résistance ciblée et résistance globale (dégradation de produits ou de publicités versus participation à des systèmes alternatifs d'échange). Les comportements étudiés incluent aussi des pratiques numériques, peu analysées dans le cadre de la résistance (pour la liste complète des pratiques étudiées, voir l'annexe).

L'objectif est de mesurer et d'analyser les pratiques de résistance sur le plan de leur catégorisation en tant que résistantes par le consommateur. Cela renvoie à la définition du comportement de résistance qui suppose une catégorisation et une intentionnalité du consommateur. La catégorisation est mesurée grâce à une échelle de Likert en 4 points (pas du tout d'accord - tout à fait d'accord).

Les données ont été analysées à l'aide de tris à plat (correspondant à l'édition de l'ensemble des modalités de réponses pour chaque question) et d'analyses bi-variées sous forme de tris croisés ou tableaux croisés faisant intervenir deux variables, la sous-population d'appartenance : digital natives versus aînés (en colonnes du tableau), et les comportements de résistance catégorisés (en lignes du tableau). Différents types de mesures d'association ont été mis en œuvre: tests de $\mathrm{khi}^{2}$, test exact de Fisher, odds ratio (produit croisé), afin de se prémunir contre les risques de surévaluation du $\mathrm{khi}^{2}$ et contre celui de distributions marginales déséquilibrées, notamment dans le cas de tableaux 2x2 (voir annexe méthodologique sur les traitements réalisés). 


\section{Une catégorisation en trois types de pratiques sous l'angle de la résistance}

L'analyse de la distribution des jugements de catégorisation sur les pratiques de résistance fait apparaitre trois niveaux :

- les pratiques qualifiées de résistantes par au moins $60 \%$ de l'échantillon («D'accord » et « Tout à fait d'accord »),

— les pratiques qualifiées de non résistantes par au moins $60 \%$ de l'échantillon («Pas d'accord » et « Pas du tout d'accord »),

- les pratiques ambivalentes, dont le caractère résistant ou non est moins consensuel, avec des taux équilibrés de réponses «D'accord» et «Tout à fait d'accord » et de réponses «Pas d'accord » et «Pas du tout d'accord » (voir annexe).

Les pratiques catégorisées comme résistantes comprennent les comportements de plainte, de boycott et de refus de la publicité, que ce soit à titre individuel ou organisé, le bouche à oreille négatif, que ce soit auprès de son entourage ou sur un réseau numérique, la contestation en mouvement organisé.

Les pratiques catégorisées comme non résistantes regroupent la dégradation de produits, le piratage et le hacking, que ce soit à titre individuel ou en mouvement collectif ainsi que l'achat de contrefaçon. Les pratiques catégorisées comme ambivalentes sont la dégradation de publicité, la copie et le téléchargement illégal, l'installation et le développement de logiciel libre, la participation à des systèmes alternatifs de consommation (troc, échange de service, monnaies alternatives). 


\section{Une césure générationnelle ? Un consensus sur la résistance mais une tolérance plus grande face à la non-résistance chez les digital natives}

Sous l'angle générationnel, l'analyse offre des résultats contrastés. Qualifiés a priori de digital natives, les jeunes diffèrent peu de leurs aînés concernant les comportements catégorisés comme résistants : les jugements sont similaires dans les deux sous-populations. Pour les comportements résistants, seules deux activités sont considérées comme significativement plus résistantes par les digital natives : la contestation organisée (85\% contre $73 \%$ ) et le refus collectif de la publicité (77\% contre $66 \%$ ). Parmi les comportements ambivalents, seul le téléchargement illégal en réseau est considéré comme significativement plus résistant par les digital natives (53\% contre $38 \%$ ). Cela est lié à un jugement plus polarisé des deux sous-populations. Les digital natives sont plus nombreux sur la modalité « Tout à fait d'accord $(19 \%$ contre $7 \%)$, tandis que les plus âgés sont plus nombreux sur la modalité «Pas du tout d'accord » $(28 \%$ contre $16 \%)$.

En revanche, les digital natives diffèrent plus clairement de leurs aînés sur les pratiques catégorisées comme non résistantes. Ils sont significativement plus nombreux à considérer comme résistantes la dégradation de produits collective ( $48 \%$ contre $31 \%$ ) ou individuelle (36\% contre $25 \%$ ), le piratage que ce soit en réseau ( $40 \%$ contre $22 \%$ ) ou à titre individuel ( $35 \%$ contre $24 \%$ ), le hacking en réseau (39\% contre $19 \%$ ) ou individuel (32\% contre $21 \%$ ). Cette différence s'exprime aussi sous la forme d'un moindre rejet de ces types de pratiques hors du territoire de la résistance, les digital natives présentant des taux de réponses «Pas du tout d'accord » plus faibles que leurs aînés. 
En synthèse, il semble donc y avoir un consensus entre les digital natives et leurs aînés sur les activités catégorisées comme résistantes et comme ambivalentes. En revanche, on observe une différence plus marquée sur les activités considérées comme non résistantes. Les activités concernées sont essentiellement reliées aux pratiques numériques avec le piratage et le hacking. Elle traduit une plus grande tolérance de la part des digital natives envers des pratiques s'apparentant à des comportements illégaux et à des atteintes plus graves aux personnes et aux biens.

\section{La résistance du consommateur : trois types de territoires}

Trois grands territoires ont pu être mis en évidence: un territoire de la résistance du consommateur aux actions et dispositifs marketing, un territoire de la non-résistance et un territoire composé de pratiques qui, selon les consommateurs, peuvent être considérées ou non comme de la résistance, un territoire qualifié d'ambivalent.

Le territoire de la résistance est composé de pratiques renvoyant essentiellement au «pouvoir d'expression » («voice »), tel qu'il a été défini par Robert O. Hirschman (1970). Il s'agit d'une forme d'expression à la fois ascendante du consommateur vers l'entreprise (plainte, boycott, contestation) et horizontale avec le bouche à oreille négatif. On constate également un rejet de la communication descendante de l'entreprise vers le consommateur à travers le refus de publicité. Ces pratiques regroupent aussi des comportements d'évitement, de rejet et de rétorsion envers les produits, marques et entreprises, à travers le boycott par exemple, qui correspondent au pouvoir de défection («exit») tel que défini par Robert O. Hirschman (ibid.), d'une part, et à une remise en cause des offres des entreprises énoncée par Lisa 
Peñaloza et Linda L. Price (1993), d'autre part. Ces pratiques sont aussi bien individuelles que collectives.

Le territoire de la non-résistance recouvre des activités illégales qui correspondent à des comportements radicaux (au sens de Lisa Peñaloza et Linda L. Price, 1993), de rejet du marketing et du marché, tels la dégradation de produits ou l'achat de contrefaçon, ou des activités illégales, comme le piratage et le hacking, que ce soit à titre individuel ou collectif.

Le territoire qualifié d'ambivalent regroupe des pratiques illicites telles la copie et le téléchargement illégal, la dégradation de publicité, des activités numériques à dimension libertaire, avec le mouvement du logiciel libre, et la participation à des systèmes alternatifs de consommation. Ces pratiques sont diverses dans leur portée et leur gravité. Elles traduisent une absence de consensus de la part des consommateurs quant à leur caractère résistant ou non. Certaines s'inscrivent dans un cadre alternatif au marché, logiciel libre et systèmes alternatifs de consommation. D'autres semblent avoir une portée plus utilitaire - copie et téléchargement illégal - ou partiellement militante, avec la dégradation de publicité ou à nouveau la copie et le téléchargement illégal.

Si l'on considère le territoire de la résistance dans un contexte numérique, on peut dissocier deux catégories de pratiques. Les activités traditionnelles d'expression («voice») telles le bouche à oreille, quand elles sont pratiquées sur un support numérique, voient leur portée amplifiée ; ce qui renvoie à la notion de territoire augmenté de Pierre Musso (2008). Elles sont catégorisées sans ambiguïté comme appartenant au territoire de la résistance. En revanche, les pratiques qui ont été rendues possibles par le numérique, logiciel libre ou téléchargement illégal, sont catégorisées comme ambivalentes, ou comme appartenant au 
territoire de la non-résistance, le piratage et le hacking. Il semble donc que les pratiques purement numériques aient encore du mal à se faire admettre dans le territoire de la résistance. Cependant, si l'on introduit la césure générationnelle, les digital natives sont plus tolérants envers l'admission de pratiques numériques telles le hacking et le piratage dans le territoire de la résistance. Ce dernier résultat donne du crédit à la métaphore de Marc Prensky sans toutefois y souscrire pleinement. Ou, pour le dire autrement, lorsque la question est posée aux jeunes nés avec le numérique, dès lors qu'elles sont numériques, les pratiques de hacking et de piratage semblent plus naturellement inscrites dans le territoire de la résistance.

\section{Conclusion}

Les résultats confirment et complètent les deux axes proposés par Lisa Peñaloza et Linda L. Price (1993). Le premier axe de leur typologie oppose comportements individuels versus collectifs. Le territoire de la résistance est composé d'activités aussi bien individuelles que collectives. Il en est de même pour le territoire de la non-résistance et le territoire qualifié d'ambivalent. La structuration des territoires ne se fait pas en fonction de l'aspect organisé ou non de la pratique, mais selon l'activité elle-même. Le clivage comportement réformiste versus radical apparaît. En effet, relèvent du territoire de la résistance les plaintes, contestations, bouche à oreille négatifs, qui renvoient à des comportements réformistes dans le cadre des institutions sociales sinon de marché. Le territoire de la non-résistance regroupe des formes plus radicales de rejet du marché et des institutions de marché telles la dégradation de produits, le piratage et le hacking. 
De plus, les résultats montrent que certaines pratiques considérées comme résistantes dans la littérature académique ne sont pas catégorisées comme telles par les consommateurs interrogés. Cette recherche explore de manière plus fine le territoire de la résistance. L'approche emic et la perspective intentionnaliste permettent de distinguer le territoire de la résistance de celui de la non-résistance. Elles font aussi émerger un territoire ambivalent englobant des pratiques caractérisées par une absence de consensus quant à leur catégorisation comme résistantes ou non. Certaines de ces pratiques sont illicites, d'autres s'inscrivent comme des alternatives au marché, d'autres enfin relèvent de l'utilitarisme ou du militantisme.

L'analyse révèle également les spécificités des pratiques numériques, catégorisées comme résistantes pour les activités traditionnelles d'expression, plus ambivalentes pour les pratiques nouvelles rendues possibles par le numérique. Par ailleurs, l'étude suggère l'existence d'une césure générationnelle quant à la catégorisation de certaines pratiques numériques. Elle montre une différence d'appréciation entre les digital natives et leurs aînés sur certains comportements ambivalents, comme le hacking et le piratage. Selon les consommateurs considérés, le devenir de ce territoire ambivalent reste à déterminer.

En outre, cette recherche confirme la grande diversité de la catégorisation des comportements de résistance de la part du consommateur à laquelle sont confrontés les acteurs du marché. Ces résultats peuvent aider les entreprises comme les institutions publiques à définir des politiques de prise en compte, de prévention ou de lutte contre ces pratiques. Les organisations, que ce soit des entreprises ou des institutions publiques, n'ont plus forcément un interlocuteur identifié (association de droits de l'homme, organisation non gouvernementale, par exemple) avec lequel elles peuvent négocier pour gérer la situation de 
crise. Elles sont confrontées à une résistance plus diffuse et floue, donc difficile à anticiper, identifier et gérer. Pour autant, grâce aux traces laissées sur les réseaux sociaux, ces organisations peuvent, à travers des outils de veille, anticiper l'émergence d'un mouvement ou d'une crise (surveiller des signaux faibles) ou même apporter une réponse en utilisant les réseaux numériques, de manière officielle ou dissimulée derrière des réseaux sociaux numériques. Plutôt que de vouloir gérer la crise, et donc le territoire numérique de résistance, elles doivent déployer une stratégie de veille et d'influence plus indirecte. L’identification des pratiques catégorisées comme résistantes permet de mieux cerner et gérer ce territoire, notamment par la mise en place d'un «zonage pertinent de capteurs d'alerte » (Boullier, $2009: 10)$.

Il convient de souligner certaines difficultés de cette recherche relevant de la méthodologie et du cadre conceptuel mobilisé. Sur le plan méthodologique, l'étude a été réalisée auprès d'un échantillon de convenance et ne peut donc prétendre à une quelconque représentativité. De même, si elle envisage une palette diversifiée de comportements et d'activités des consommateurs, elle ne peut prétendre à l'exhaustivité. Enfin, les mesures utilisées reposent sur des échelles ad hoc dont les propriétés psychométriques ne sont pas établies. Sur le plan conceptuel, le concept de digital natives a été opérationnalisé par Marc Prensky sous la forme d'une césure générationnelle fondée sur l'âge. Il conviendrait de mettre en relation ce concept avec d'autres éléments tels le genre ou les critères sociaux, dans le cadre d'une étude mobilisant un échantillon représentatif de la population de référence. On peut aussi s’interroger sur l'homogénéité des catégories proposées par Marc Prensky et explorer plus avant la diversité à l'intérieur des deux groupes. Un autre prolongement de la recherche pourrait être la focalisation sur certains types d'activités peu étudiés, comme les activités numériques, ou sur des segments spécifiques, tels les Amapiens ou les hackers et pirates en 
réseau. Ceci permettrait d'éclairer et d'expliquer les perceptions différenciées et les clivages sous-jacents, et d'intégrer une dimension de temporalité.

\section{Références}

Alliès P., 1980, L'Invention du territoire, Grenoble, Presses universitaires de Grenoble.

Badouard R., 2013, «Les mobilisations de clavier. Le lien hypertexte comme ressource des actions collectives en ligne », Réseaux. Communication, technologie, société, 181, pp. 87-117.

Boullier D., 2009, « Au-delà des territoires numériques en dix thèses », pp. 1-15, in : Rowe F., dir., Sociétés de la connaissance et prospective : hommes, organisations et territoires, Nantes, Université de Nantes/Conseil général des Pays de la Loire.

Capelli S., Legrand P., Sabadie W., 2012, «Se taire, nier ou s'excuser : comment répondre à un appel au boycott », Décisions Marketing, 68, oct.-déc., pp. 71-82.

Cefaï D., 2016, «Publics, problèmes publics, arènes publiques... Que nous apprend le pragmatisme », Questions de communication, 30, pp. 25-64.

Chalamon I., Chouk I., Guiot D., 2012, « La cyber-résistance du consommateur : quels enjeux pour les entreprises », Décisions Marketing, 68, oct.-déc., pp. 83-88.

Cho C. H., Cheon H. J., 2004, «Why Do People Avoid Advertising on the Internet? », Journal of Advertising, 33 (4), pp. 89-97.

Cissé-Depardon K., 2009, «Les déterminants individuels de la décision de participation à un boycott », pp. 159-178, in : Roux D., dir., Marketing et résistance(s) du consommateur, Paris, Éd. Economica. 
Cissé-Depardon K., N'Goala G., 2009, «Les effets de la satisfaction, de la confiance et de l'engagement vis-à-vis d'une marque sur la participation des consommateurs à un boycott », Recherches et applications en marketing, 24 (1), pp. 43-67.

Cottet P., Ferrandi J. M., Lichtle M. C., 2009, «La résistance des consommateurs à la publicité », pp. 129-144, in: Roux D., dir., Marketing et résistance(s) du consommateur, Paris, Éd. Economica.

Cottet P., Ferrandi J. M., Lichtle M. C., 2012, «Les consommateurs résistants à la publicité : leurs principales actions et motivations », Décisions Marketing, 68, oct.déc., pp. 25-36.

Cox J., Collins A., Drinkwater S., 2010, «Seeders, Leechers and Social Norms: Evidence from the Market for Illicit Digital Downloading », Information Economics and Policy, 22, pp. 299-305.

Dalli D., Gistri G., Romani S., 2005, «Brand Dislike: Evidence from Qualitative Research and Scale Development. Rejuvenating Marketing: Contamination, Innovation, Integration », $34^{\text {th }}$ European Marketing Academy Conference, Milan, mai.

Dobscha S., Ozanne J. L., 2001, «An Ecofeminist Analysis of Environmentally Sensitive Women Using Qualitative Methodology: Facing the Emancipatory Potential of Ecological Life », Journal of Public Policy and Marketing, 20 (2), pp. 201-214.

Duke L., 2002, «Get real! Cultural Relevance and Resistance to the Mediated Feminine Ideal », Psychology and Marketing, 19 (2), pp. 211-233.

Fontaine I., 2009, «La résistance du consommateur au placement des marques dans les films », pp. 145-158, in: Roux D., dir., Marketing et résistance(s) du consommateur, Paris, Éd. Economica.

Fournier S., 1998, «Consumer Resistance: Societal Motivations, Consumer Manifestations, and Implications in the Marketing Domain », Advances in Consumer Research, 25, pp. 88-90. 
Friedman M., 1985, «Consumer Boycotts in the United States, 1970-1980: Contemporary Events in Historical Perspective », The Journal of Consumer Affairs, 19 (1), pp. 96-117. Garcia-Bardidia R., Nau J. P., Rémy E., 2011, «Consumer Resistance and AntiConsumption. Insights from the Deviant Careers of French Illegal Downloaders », European Journal of Marketing, 45 (11-12), pp. 1789-1798.

Garcia-Bardidia R., Nau J. P., Rémy E., 2012, «La consommation illégale de musique numérique : y résister ou se l'approprier ? », Décisions Marketing, 68, oct.-déc., pp. 8994.

Granjon F., 2017, «Résistances en ligne : mobilisation, émotion, identité », Variations, 20, pp. 1-24.

Guiton A., 2013, Hackers. Au cœur de la résistance numérique, Paris, Éd. Le Diable Vauvert. Herrmann R. O., 1993, «The Tactics of Consumer Resistance: Group Action and Market Place Exit », Advances in Consumer Research, 20, pp. 130-134.

Hirschman A. O., 1970, Exit, Voice, and loyalty. Responses to declines in firms, organization and states, Cambridge, Harvard University Press.

Jones C., Shao B., 2011, The Net Generation and digital natives - Implications for higher education Academy, York, Higher Education Academy.

Kelly L., Kerr G., Drennan J., 2010, « Avoidance of Advertising in social Networking Sites: The Teenage Perspective », Journal of Interactive Advertising, 10 (2), pp. 16-27.

Kerr G., Mortimera K., Dickinson S., Waller D. S., 2012, «Buy, Boycott or Blog - Exploring Online Consumer Power to Share, Discuss and Distribute Controversial Advertising Messages », European Journal of Marketing, 46 (3-4), pp. 387-405.

Kirmani A., Campbell M., 2004, «Goal Seeker and Persuasion Sentry: How Consumer Targets Respond to Interpersonal Marketing Persuasion », Journal of Consumer Research, 31 (3), pp. 573-582. 
Krishnamurthy S., Kucuk S. U., 2009, «Anti-Branding on the Internet », Journal of Business Research, 62, pp. 1119-1126.

Le Roux A., Stenger T., Thébault M., 2015, « Les digital natives sont-ils des consommateurs plus résistants ? », in: Stenger T., dir., Digital Natives - Culture, génération et consommation, Cormelles-le-Royal, EMS Éd.

Moisio R., Askegaard S., 2002, «Fighting Culture. Mobile Phone Consumption Practices as Means of Consumer Resistance », Asia Pacific Advances in Consumer Research, 5, pp. 24-29.

Musso P., 2008, « Territoires numériques », Medium, 15, pp. 25-38.

Musso P., 2009, «Critique de la notion de territoire numérique », pp. 168-175, in : Gille L., dir., Les Dilemmes de l'économie numérique, Limoges, FYP Éd.

Odou P., Bonnin G., 2014, «Les stratégies de neutralisation de la pression normative par les consommateurs: le cas du téléchargement illégal », Recherche et applications en marketing, 29 (1), pp. 114-133.

Olivier De Sardan J.-P., 1998, «Émique », L'Homme. Revue française d'anthropologie, 147, pp. 151-166.

Palfrey J., Gasser U., 2008, Born Digital. Understanding the first generation of digital natives, New York, Basic.

Peñaloza L., Price L. L., 1993, «Consumer Resistance: A Conceptual Overview », Advances in Consumer Research, 20, pp. 123-128.

Pez V., 2012, «Comprendre les comportements de rejet de certains consommateurs face aux programmes de fidélité », Décisions Marketing, 68, oct.-déc., pp. 37-46.

Prensky M., 2001a, « Digital Natives, Digital Immigrants », On the Horizon, 9 (5), pp. 1-6.

Prensky M. 2001b, «Digital Natives, Digital Immigrants, part 2: Do They Really Think Differently? », On the Horizon, 9 (6), pp. 1-6. 
Prensky, M., 2009, «H. Sapiens Digital: From Digital Immigrants and Digital Natives to Digital Wisdom», Innovate. Journal of Online Education, 5 (3). Accès : https://nsuworks.nova.edu/innovate/vol5/iss3/1/.

Prensky M., 2010, Teaching Digital Natives. Partnering for Real Learning, Londres, Sage.

Prensky M., 2015, « Digital natives, technologie et culture numériques - Entretien avec Marc Prensky », pp. 25-35, in: Stenger T., dir., Digital Natives - Culture, génération et consommation, Cormelles-le-Royal, EMS Éd.

Robert-Demontrond P., 2009, «Une micro-mythanalyse de l'imaginaire des AMAPiens », pp. 107-128, in : Roux D., dir., Marketing et résistance(s) du consommateur, Paris, Éd. Economica.

Roux D., 2007, «La résistance du consommateur : proposition d'un cadre d'analyse », Recherche et applications en marketing, 22 (4), pp. 55-80.

Roux D., 2008, «Consumers Faced With Telephone Selling: Metacognition, Resistance and Strategies », Advances in Consumer Research, 35, pp. 467-474.

Roux D., 2009, Marketing et résistance(s) du consommateur, Paris, Éd. Economica.

Roux D., 2012, «Au-delà des réclamations : comprendre et gérer les litiges », Décisions Marketing, 67, juil.-sept., pp. 49-62.

Roux D., 2014, Présentation journée de recherche Cerege, 6 mai, Poitiers, Institut d'administration des entreprises de Poitiers.

Rumbo J. D., 2002, «Consumer Resistance in a World of Advertising Clutter: The Case of Adbusters », Psychology and Marketing, 19 (2), pp. 127-148.

Serres M., 1997, Le Parasite, Paris, Hachette.

Shaw D., Newholm T., 2002, «Voluntary Simplicity and the Ethics of Consumption », Psychology and Marketing, 19 (2), pp. 167-185. 
Sitz L., 2009, «Lier les résistances: au-delà de la dichotomie entre l'individuel et le collectif », pp. 89-105, in: Roux D., dir., Marketing et résistance(s) du consommateur, Paris, Éd. Economica.

Stenger T., 2015, Digital Natives - Culture, génération et consommation, Cormelles-leRoyal, EMS Éd.

Thébault M., 1999, Le Boycott : analyse conceptuelle et modélisation, Thèse de doctorat en sciences de gestion, Saint-Denis, Université de la Réunion.

Thomas M., 2011, Deconstructing Digital Natives, Londres, Routledge.

Thompson C. J., Haytko D. L., 1997, «Speaking of Fashion: Consumers’ use of Fashion Discourses and the Appropriation of Countervailing Cultural Meanings », Journal of Consumer Research, 24 (1), pp. 15-42.

Urbain C., Le Gall-Ely M., Urien B., 2012, «Les associations doivent-elles craindre une résistance au don d'argent ? Premières pistes conceptuelles et managériales », Décisions Marketing, 68, oct.déc., pp. 59-70.

Zavestoski S., 2002, «The Social-Psychological Bases of Anticonsumption Attitudes », Psychology and Marketing, 19 (2), pp. 149-165.

\section{Annexe}

Catégorisations par les enquêtés (290) des pratiques considérées par la littérature scientifique comme relevant de la résistance du consommateur aux actions et dispositifs marketing

\begin{tabular}{|l|l|l|l|l|l|l|}
\hline & Pas du & Pas & Total & D'acco & Tout à & Total \\
Catégorisations des pratiques & tout & d'accor & en & rd & fait & en \\
\hline
\end{tabular}




\begin{tabular}{|c|c|c|c|c|c|c|}
\hline & $\begin{array}{l}\text { d'accor } \\
\text { d } \\
(\text { en } \%)\end{array}$ & $\begin{array}{l}\text { d } \\
(\mathrm{en} \%)\end{array}$ & $\begin{array}{l}\text { désacc } \\
\text { ord } \\
\text { (en \%) }\end{array}$ & $($ en \%) & $\begin{array}{l}\text { d'accor } \\
\text { d } \\
\text { (en \%) }\end{array}$ & $\begin{array}{l}\text { accord } \\
\text { (en \%) }\end{array}$ \\
\hline Comportements résistants & & & & & & \\
\hline Plainte individuelle & 10,0 & 9,0 & 19,0 & 41,0 & 39,3 & 80,3 \\
\hline Plainte collective & 5,2 & 11,0 & 16,2 & 38,3 & 45,2 & 83,4 \\
\hline Boycott individuel & 9,7 & 10,7 & 20,3 & 33,1 & 43,4 & 76,6 \\
\hline Boycott collectif & 8,3 & 9,7 & 17,9 & 41,7 & 39,0 & 80,7 \\
\hline Bouche à oreille négatif (entourage) & 9,3 & 16,2 & 25,5 & 35,2 & 37,9 & 73,1 \\
\hline Bouche à oreille négatif (blog) & 9,3 & 15,2 & 24,5 & 37,2 & 36,9 & 74,1 \\
\hline Refus de publicité individuel & 17,9 & 17,2 & 35,2 & 36,9 & 25,9 & 62,8 \\
\hline Refus de publicité collectif & 10,0 & 17,2 & 27,2 & 44,5 & 27,2 & 71,7 \\
\hline Contestation organisée & 6,6 & 13,8 & 20,3 & 34,5 & 44,1 & 78,6 \\
\hline Comportements non résistants & & & & & & \\
\hline
\end{tabular}




\begin{tabular}{|c|c|c|c|c|c|c|}
\hline Dégradation de produits individuelle & 37,9 & 30,3 & 68,3 & 19,3 & 11,0 & 30,3 \\
\hline Dégradation de produits collective & 29,7 & 29,0 & 58,6 & 24,5 & 15,5 & 40,0 \\
\hline Piratage individuel & 35,9 & 33,4 & 69,3 & 18,6 & 11,4 & 30,0 \\
\hline Hacking individuel & 41,4 & 31,0 & 72,4 & 15,9 & 10,7 & 26,6 \\
\hline Piratage en mouvement organisé & 32,8 & 34,8 & 67,6 & 21,0 & 10,3 & 31,4 \\
\hline Hacking en mouvement organisé & 38,3 & 31,4 & 69,7 & 19,0 & 10,3 & 29,3 \\
\hline Achat de contrefaçons & 26,9 & 32,1 & 59,0 & 22,8 & 13,8 & 36,6 \\
\hline Comportements ambivalents & & & & & & \\
\hline Copie illégales individuelles & 26,6 & 29,0 & 55,5 & 27,6 & 15,9 & 43,4 \\
\hline Téléchargement illégal individuel & 25,5 & 31,0 & 56,6 & 25,5 & 16,6 & 42,1 \\
\hline Copies illégales en réseau & 21,0 & 33,4 & 54,5 & 32,1 & 12,8 & 44,8 \\
\hline Téléchargement illégal en réseau & 21,0 & 32,8 & 53,8 & 32,1 & 13,4 & 45,5 \\
\hline Installation de logiciels libres & 24,1 & 27,9 & 52,1 & 24,5 & 22,1 & 46,6 \\
\hline
\end{tabular}




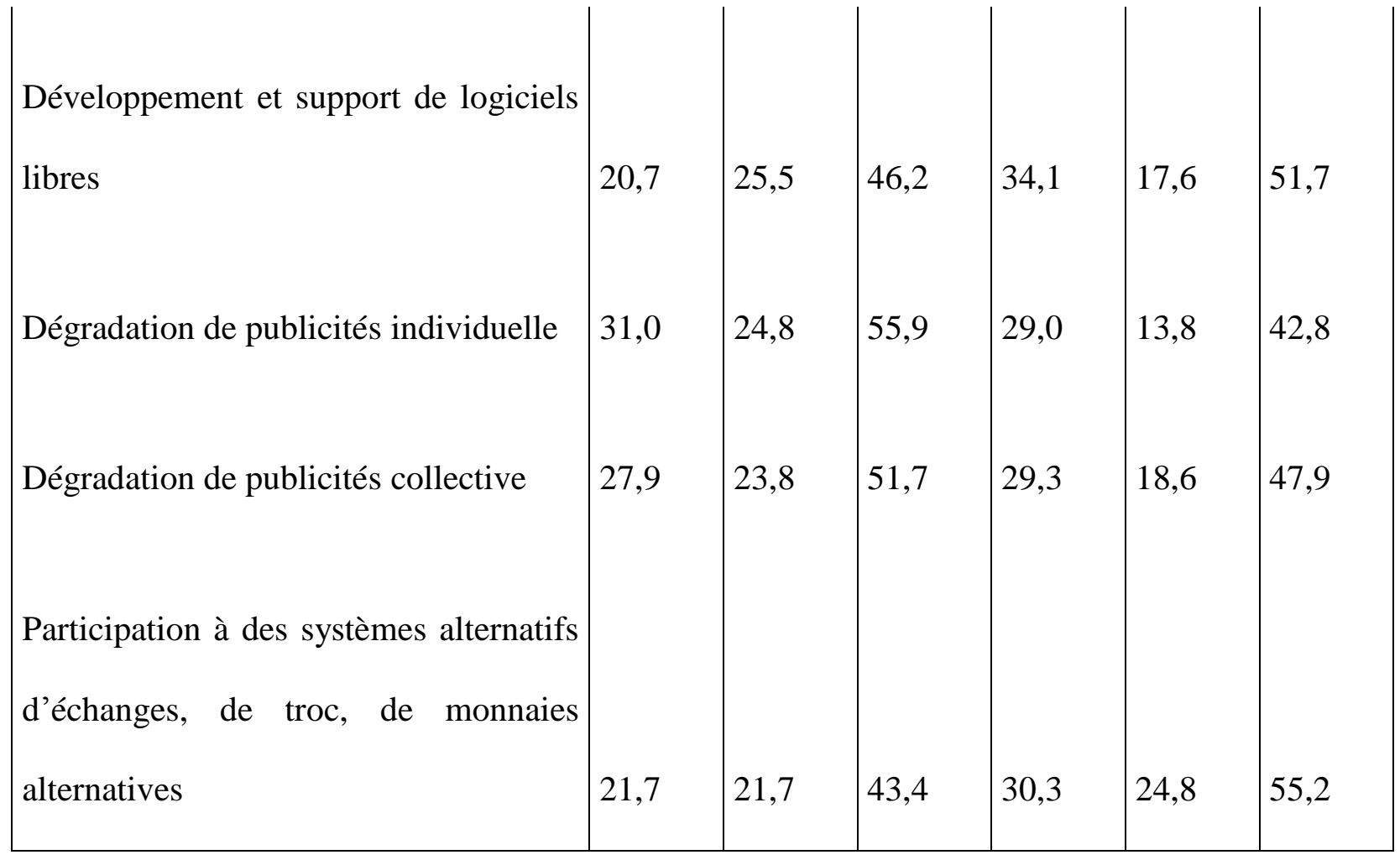

\section{Notice méthodologique sur les traitements réalisés}

L'association (ou covariation) entre la catégorisation des comportements de résistance et l'appartenance à la sous-population digital natives ou aînés a été analysée à l'aide de tests de $\mathrm{khi}^{2}$ et d'odds ratio (ou produit croisés). Le test de $\mathrm{khi}^{2}$ a été choisi car il ne suppose aucune loi de distribution théorique des données (loi normale ou autre), et peut donc être utilisé quel que soit le type de données collectées. Ce test est néanmoins sensible au risque de surévaluation de la statistique de $\mathrm{khi}^{2}$ dans le cas de tableaux $2 \times 2$ (à deux lignes et deux colonnes). Le test exact de Fisher qui vient corriger ce risque dans le cas des tableaux $2 \times 2$ a donc été utilisé. De même, le test de $\mathrm{khi}^{2}$ est sensible aux distributions marginales déséquilibrées (catégorisation d'un comportement comme non résistant par $90 \%$ ou plus des répondants, et donc comme résistant par $10 \%$ ou moins des répondants, par exemple). Un fort déséquilibre dans les distributions marginales peut conduire à ignorer une relation entre la 
sous-population d'appartenance (digital natives ou aînés) et la catégorisation d'une pratique comme résistante ou non. L'odds ratio (ou produit croisé) a donc été utilisé afin de se prémunir contre ce risque. 